The sudden appearance last year² of strains of gonococci which have acquired the ability to produce a $\beta$-lactamase (penicillinase) and so become completely resistant to penicillin and ampicillin has serious implications for the control of gonorrhoea. These strains were first seen in the United States, mainly in servicemen returning from the Far East, and in an apparently unconnected outbreak of infections on Merseyside. ${ }^{3}$ They have since been identified in at least 11 countries. ${ }^{1}$

Both Roberts and Falkow. and Eisenstein et $a^{6}{ }^{6}$ have shown that this new type of resistance is mediated by plasmidsextrachromosomal loops of DNA. Almost all gonococci contain a plasmid of molecular weight $2 \cdot 5 \cdot 10^{6}$, and some a larger one of $24 \cdot 5 \cdot 10^{6}$. Strains from the USA and Far East producing (3-lactamase contain an additional plasmid of molecular weight $4 \cdot 4 \cdot 10^{6}$ and similar strains from the Liverpool outbreak one of molecular weight $3 \cdot 2 \cdot 10^{6}$; both of these carry the genetic information coding for -lactamase production. The difference in molecular weight of these two $\mathrm{R}$ plasmids suggests that the USA Far East and Liverpool strains have originated separately. The Far Eastern strains producing ;-lactamase differ among themselves in their nutritional requirements and chromosomal antibiotic resistance genes; this suggests that in this area there has been a considerable dissemination of the $\mathrm{R}$ plasmid among different strains of gonococci rather than the spread of a single resistant strain in the population.

Transformation has not proved possible in vitro of sensitive gonococci to 5 -lactamase production by DNA from lactamase producers. Transfer seems to be due to conjugation between gonococci, and probably only those strains having the large $24.510^{6}$ plasmid can act as donors. Hence this plasmid may have a sex factor activity and transfer of the smaller resistance plasmid is promoted during conjugation. Other findings suggest that this large plasmid may promote transfer of chromosomal genes; ; if that is confirmed the emergence of multiply resistant penicillinase-producing strains will be a possibility. The large sex factor plasmid was found in three of the nine Far Eastern strains but in none of the three Liverpool strains studied. In mating experiments Eisenstein and his colleagues" were able to transfer the ability to produce " $\%$ lactamase from gonococci to Escherichia coli and Neisseria flava. This has serious implications; the possibility of transfer in vivo to meningococci is especially worrying.

Where did the first - -lactamase producing gonococcus get its $\mathrm{R}$ plasmid from and why has it taken so long to do so ? Percival et $\mathrm{al}^{3}$ found that the substrate profile of the Liverpool strains was similar to that of an $E$ coli TEM $\%$-lactamase, and in isoelectric focusing experiments both enzymes gave a band focusing at $\mathrm{pH} 5 \cdot 4$. Rectal lesions are common in women with gonorrhoea, and $E$ coli and gonococci must have coexisted in this site on innumerable occasions. Hatmophilus influenzae may also have been responsible for this unwelcome gift. Plasmidmediated $p$-lactamase producing strains of haemophilus have emerged during the past few years. Pharyngeal gonorrhoea transmitted by orogenital sexual practices provides ample opportunity for these two organisms to meet.

The potential threat which penicillinase-producing gonococci pose to the control of gonorrhoea is obvious, but it should be kept in perspective. In Britain the outbreak on Merseyside seems to have been contained: only a handful of strains have been seen in other parts of the country, most of which originated from infections in the Liverpool area. This is a tribute to the efficiency of the contact tracing methods used there, and these form the major method for containment. Screening gonococcal isolates for sensitivity to penicillin by a simple disc method will rapidly identify strains needing further investigation; full sensitivity studies are also essential in all patients failing to respond to penicillin. Spectinomycin or cefuroxime are effective for treating patients known to be infected with -lactamase producing gonococci or their contacts.

If the prevalence of "-lactamase producing strains in Britain remains as low as it is now there is no need to abandon the use of penicillin or ampicillin. In high prevalence areas, such as the Philippines-where $20-40^{\circ}{ }_{0}$ of prostitutes are said to carry these strains ${ }^{i}$ - a reappraisal of treatment methods is clearly needed. But with the global distribution of these strains and the speed of air travel inevitably further resistant strains will be imported into Britain. We need to identify and eradicate them before they can establish themselves in the community.

\footnotetext{
' Sparling, P F, et al, in International Symposium on Gonorrhoea, p 92 Ottawa, Information Canada, 1975.

- British Medical fournal, 1976, 2, 963.

3 Percival, A, et al, Lancet, 1976, 2, 1379.

WHo Weekly Epidemiological Record, 1976, 51, 385.

Roberts, M, and Falkow, S, Nature, 1977, 266, 630.

"Eisenstein, B I, et al, Science, 1977, 195, 998.

- Culliton, B J, Science, 1976, 194, 1395.
}

\section{Polyamines as markers of response to chemotherapy of cancer}

The polyamines putrescine, spermidine, and spermine are small cations now thought by some research workers to be vital as regulators of tissue growth. This seems a long way from earlier ideas in which putrescine was considered a product of bacterial decay of mammalian tissue and spermidine and spermine were thought simply to be degradation products excreted into seminal fluid. In fact polyamines and their biosynthetic enzymes are ubiquitous. ${ }^{1}$ The increase of RNA in cells as they grow and reproduce is paralleled by an increase of spermidine and preceded by a build up of putrescine. In mammalian tissues it is the decarboxylation of ornithine that is the source of putrescine, which in turn yields spermidine and spermine. The enzyme ornithine decarboxylase has an unusually high turnover rate in mammalian cells; its level is normally quite low but rises rapidly in response to growth stimuli.

At this point the story passes on to weaker ground. While it can be shown that polyamines have an affinity for nucleic acids and can stimulate protein synthesis both in vivo and in vitro, no specific mechanism has been firmly established for the action of polyamines in vivo: and many biochemists are trying to determine the part that these simple molecules play in controlling nucleic acids and the synthesis of proteins. At present it seems that if they are concerned in the regulation of tissue growth then they behave in a different fashion from other forms of well-established growth regulators, for their concentrations in cells are far too high according to current biochemical theory of the action of regulators. Furthermore, though the activities of ornithine decarboxylase can change quickly, such a mechanism is still comparatively slow in terms of the speed of biochemical reactions.

Diane Russell has argued strongly that these compounds deserve more attention as biochemical markers of cancer and has recently reviewed the evidence. Techniques for isolating 
and measuring polyamines are still being refined, and earlier results were often plagued with erroneous measurements. But the current prospects for developing sensitive, accurate polyamine assays are good, ${ }^{2}$ and the work so far done is therefore well worth examination.

Increased amounts of polyamines in the urine of patients with cancer were reported in $1971 .^{3}$ Since then the observation has been confirmed but its practical value has been less obvious. Recently, however, Durie et $a l^{4}$ analysed polyamine excretion patterns in 124 patients with haematological and solid tumours, looking closely at the levels of polyamine excretion in relation to disease activity and response to chemotherapy. They showed that the ratio between amounts of urinary spermidine output before and after treatment correlated with the response to chemotherapy. In patients who made a complete or partial response to treatment this ratio increased nearly fourfold, but it never increased more than twofold in the non-responders. The size of the overall tumour mass also influenced the amounts of urinary spermidine in the untreated patients, and the more aggressive forms of tumours produced a higher output of polyamines than the slowly progressive forms of the same type of cancer.

At about the same time as these results were published Seymour Cohen" reported the conclusions of an American Cancer Society Workshop meeting on polyamines in cancer. Polyamines were thought to have little part in cancer screening and, with few exceptions, probably not to add much as an aid to diagnosis. On present evidence polyamines seemed most promising in the short-term evaluation of the efficacy of treatment: a transient rise in the urinary and blood polyamine concentrations for the few days after a course of chemotherapy for leukaemia and solid tumours indicated a good response. In long-term surveillance polyamine levels seemed to correlate with clinical state, and a rise of putrescine may precede a decline in clinical state. Overall, this report emphasised that careful studies on the origin of polyamines in patients with cancer would be needed before the true relevance of their apparent correlation with tumour burden could be decided.

Here, then, is a complex area of biochemistry in which concentrations in body fluids of three related variantsspermine, spermidine, and putrescine-may change in response to tissue proliferation and necrosis due to cancer or other causes. The disentanglement of their relationships will require more work in carefully staged disease as well as horizontal sequential studies to discern their changing patterns with the evolution of cancer in the same patient. As with other tumour-associated reactions (such as the modification of the profile of acute-phase reactant proteins) research will require a combination of precise chemical measurement, sound clinical assessment, and the application of the advanced statistical techniques of multivariate analysis.

1 Tabor, C W, and Tabor, H, Annual Reviea of Biochemistry, 1976, 45, 285

2 Russell, D H, Clinical Chemistry, 1977, 23, 22.

3 Russell, D H, Nature New Biology, 1971, 233, 144.

4 Durie, B G M, Salmon, S E, and Russell, D H, Cancer Research, 1977, 37, 214.

5 Cohen, S S, Cancer Research, 1977, 37, 939.

\section{Change in New England}

Ten years ago, at the time when Dr Franz Ingelfinger became editor of the New England Fournal of Medicine, there were many who doubted that general medical journals would survive into the last quarter of the century. Had not Sir Theodore Fox, that most respected name in medical editing, predicted $^{1}$ that journals such as the Lancet had had their day, and that they would split into two subspecies, medical recorders and medical newspapers?

Yet in 1977 the major weekly journals are healthier than ever, with rising circulations and enthusiastic readers. A balance has been struck between the need to evaluate scientific papers and the maintenance of newsworthiness; Dr Ingelfinger has recently described his own approach in his Shattuck lecture, ${ }^{2}$ whose title, "The General Medical Journal: for Readers or Repositories," summed up the editorial dilemma. The decade of his editorship has been one of continued, growing success for the NEFM. Perhaps the most noticeable change in that time has been the development of its correspondence columns into a forum for vigorous debate. Another notable feature has been his willingness to publish outspoken articles on politically sensitive topics such as the story of medicine and the coup in Chile-and his recent courageous attack on the quack cancer-cure laetrile. ${ }^{3}$ We shall all be sad that Dr Ingelfinger is to hand over the editor's chair. His successor is Dr Arnold S Relman, former editor of the Fournal of Clinical Investigation, who is no stranger to the NEFM, having served on the Committee on Publications of the Massachusetts Medical Society. All friends of the NEFM will wish him well in the challenging task of taking over from a fine and respected editor.

${ }^{1}$ Fox, T, Crisis in Communication: The functions and future of medical journals. London, Athlone Press, 1965.

Ingelfinger, F J, New England fournal of Medicine, 1977, 296, 1258.

${ }^{3}$ Ingelfinger, F J, New England fournal of Medicine, 1977, 296, 1167. 